Ann. Biol. anim. Bioch. Biophys., 1978, 18 (1), 21-27.

\title{
Cartilage de croissance dans ses aspects métaboliques et la régulation hormonale de son développement
}

\author{
par R. RAPPAPORT, Marie-Thérèse CORVOL \\ Unité de Recherche sur les Maladies du Rein ef du Méfabolisme, chez l'Enfant, INSERM U 30, \\ Hôpital des Enfants Malades, 149, rue de Sèvres 75730 Paris Cedex 15.
}

Summary. The metabolic aspects of growth plate cartilage and the hormonal regulation of its development.

The epiphyseal growth plate plays a major role in skeletal growth. Endocrine factors regulate the process of cell multiplication and maturation, as well as the specific metabolic activity of that organ, i.e. formation of collagen and proteoglycans. The present report is a review of the most usual methods used to investigate the metabolic activity of cartilage. Besides chemical studies and incubation techniques with different types of cartilage, chondrocyte culture provides new methods of investigation. Articular and growth plate chondrocytes can be compared according to their ability to develop in cultures, to produce specific proteoglycans and respond to hormones. New data were found demonstrating the stimulatory action of somatomedin peptides and the active metabolites of vitamin $D_{3}$ on sulfate uptake into proteoglycans. Other studies in vitro showed that cortisol was inhibitory at physiological concentrations, and that triiodothyronin, and to a much lesser extent thyroxin, stimulated the sulfate uptake. No direct testosterone or estradiol action could be demonstrated. Little is known about the mode of hormonal action on the chondrocytes or the epiphyseal cartilage. Some hormones might interfere with the somatomedin action at the cellular level. A careful evaluation of the in vitro action of hormones on cartilage at low concentrations is needed.

La croissance staturale dépend d'un processus d'ossification enchondrale au niveau des os longs et du rachis. Cette ossification se distingue des autres modes de formation osseuse tels l'ossification du crâne et de la face ou le phénomène général de renouvellement osseux. Elle est en relation avec l'activité du cartilage de croissance qui détermine la capacité d'allongement du squelette. Ce cartilage constitue un organe particulier par la très longue persistance d'une capacité à la multiplication cellulaire suivie d'une séquence d'événements comportant la maturation des chondrocytes, la minéralisation de la matrice cartilagineuse, enfin la formation de l'os. La régulation de cette activité est liée à des facteurs génétiques, vasculaires, mécaniques, métaboliques ef hormonaux. 


\section{Structure et métabolisme du cartilage de croissance.}

Après la naissance, l'épiphyse des os longs est revêtue d'un cartilage articulaire qui protège la partie sous jacente destinée à devenir le cartilage de croissance. Entre ces deux types de cartilage, il existe des différences importantes, qui portent sur la nature des protéoglycannes et surtout sur l'arrangement et le processus de maturation cellulaire. Ainsi, le cartilage de croissance se divise en 4 zones : La zone de réserve où les chondrocytes sont au repos ; la zone de prolifération où les chondrocytes se multiplient ef s'empilent en colonnes parallèles ; la zone de maturation où les chondrocytes ne se multiplient plus, mais augmentent de volume ; la zone d'hypertrophie et de dégénérescence où les chondrocytes se lysent ef où commence la ligne de calcification provisoire.

Dans ce tissu complexe, un seul et même type cellulaire acquiert des fonctions métaboliques différentes suivant sa localisation. Le contenu extracellulaire ou matrice du cartilage varie lui aussi suivant les zones considérées. Il est formé de fibres de collagène de Type II (différentes des fibres de collagène de Type I osseux), de glycoprotéines et de protéoglycannes soufrés. Ces derniers sont des macromolécules formées de nombreuses chaînes dissacharidiques fixées par des liaisons covalentes à une protéine porteuse, par l'intermédiaire de la sérine ou de la thréonine. Ces chaînes dissacharidiques ou glycosaminoglycannes sont soufrées (chondroitine-4-sulfate, 6-sulfate et kératane sulfate). Les protéoglycannes sont formés de subunités qui s'associent de façon non covalente par l'intermédiaire de l'acide hyaluronique et de deux protéines de liaison pour former des agrégats de poids moléculaires très élevés. Dans la zone de prolifération les fibres de collagène orientées entre les colonnes cellulaires sont liées aux protéoglycannes arrangés sous forme d'agrégats. Par contre, dans la zone de maturation puis dans la zone hypertrophique, les protéoglycannes se trouvent essentiellement sous formes de subunités (Hardingham and Muir, 1972 ; Gregory, 1973). C'est dans cette zone que la calcification provisoire va commencer. Dans le développement de celle-ci, les rôles respectifs des protéoglycannes, du collagène, de l'activité phosphatasique des chondrocytes, et des lipides, ne sont pas encore précisés. Cette synthèse complexe des protéoglycannes se situe au niveau de plusieurs structures intracellulaires. La portion protéique est synthétisée dans le réticulum endoplasmique rugueux. II est possible que les chaînes de glycosaminoglycannes soient aussi amorcées à ce niveau. Ce matériel est transféré au niveau de l'appareil de Golgi où les chaînes latérales subissent un allongement ef la sulfatation. Finalement, ces protéoglycannes sont sécrétés par un mécanisme probable d'exocytose vers la matrice intercellulaire. Par ailleurs, les axes vasculaires venus de la zone osseuse s'insinuent au contact des cellules hypertrophiques dégénérées. Le rôle de l'oxygénation et de la vascularisation n'est certainement pas négligeable à ce niveau.

\section{Méthodes d'études.}

Les études métaboliques portent habituellement sur la multiplication cellulaire et la synthèse protéique spécifique. Ces deux phénomènes ont lieu simultanément au cours du développement du cartilage de croissance mais avec une prédominance du 
premier dans la zone des colonnes alors que les synthèses protéiques sont prédominantes dans la zone de maturation.

La mesure de la multiplication cellulaire peut se faire en comptant les cellules présentes, en mesurant le DNA pondéral, en mesurant l'incorporation de thymidine tritiée dans les cellules par autoradiographie ou après extraction du DNA.

La mesure des synthèses protéiques peut porter sur l'incorporation d'un acide nucléique tel l'uridine ou l'incorporation d'un acide aminé marqué non spécifique tel la ${ }^{14} \mathrm{C}$-leucine. Contrairement au tissu osseux où la synthèse protéique spécifique prépondérante est celle du collagène, le cartilage de croissance contient essentiellement des protéoglycannes soufrés. C'est la mesure de l'incorporation de sulfate ${ }^{35}$ S dernier temps de cette synthèse qui est utilisée par la plupart des auteurs. L'incorporation de sulfate peut être mesurée dans les chaînes de glycosaminoglycannes séparées de la chaîne proféique après action de la pronase, ou dans les protéoglycannes entiers extraits et purifiés sous forme de subunités.

L'effet des hormones polypeptidiques sur le système AMP-cyclique peut se traduire par des variations de concentration d'AMP-cyclique. Celle-ci peut être liée à une activation de l'adénylcyclase et plus rarement de l'activité phosphodiestérasique.

Ces activités métaboliques peuvent être étudiées sur des fragments de cartilage, des cellules isolées après digestion enzymatique (Garland, 1972) ou des cellules mises en culture. Ce dernier modèle a été récemment développé pour des chondrocytes du cartilage de croissance tibial de lapin (Corvol ef al., 1975) selon une technique dérivée de celle qui était utilisée pour les chondrocytes d'origine articulaire (Green, 1973). Ces chondrocytes de la zone proliférative du cartilage de croissance de lapin conservent leur différenciation dans des conditions particulières de culture (Corvol et al., 1974). Ils se multiplient en colonnettes et non en monocouches à la différence des chondrocytes articulaires (Corvol et al., 1975). Les protéoglycannes soufrés synthétisés dans leur milieu de culture sont purifiés sur colonne de DEAE cellulose et par migration sur gel d'acrylamide-agarose. Leurs propriétés électrophorétiques sont similaires à celles de protéoglycannes extraits du même cartilage de croissance in vivo mais différentes de celles des protéoglycannes provenant de cartilage articulaire ou de chondrocytes articulaires mis en culture. Ces macromolécules sont sécrétées dans le milieu extracellulaire et jouent encore un rôle dans la régulation de la synthèse intra-cellulaire.

\section{Effets des hormones sur le cartilage.}

Seuls les effets directs des hormones, tels qu'ils peuvent être observés in vitro, seront décrits (tabl. 1).

Les peptides à activités somatomédine isolés à partir du plasma humain. Il est démontré depuis longtemps (Salmon et Daughaday, 1957) que l'hormone de croissance n'a pas d'effet direct sur le cartilage. Par contre, elle provoque l'apparition dans le sérum normal, d'un facteur de sulfatation, appelé encore activité somatomédine (Daughaday et al., 1972). Il est probable que cetfe activité stimulante au niveau du cartilage, est aussi liée à d'autres hormones circulantes en particulier stéroïdes et thyroïdiennes. 
L'action directe de ces dernières au niveau du cartilage commence seulement à être décrite.

TABLEAU 1

Etudes in vitro sur l'action des hormones au niveau du cartilage

\begin{tabular}{|c|c|c|}
\hline Hormone & Type de cartilage & Effet sur le cartilage \\
\hline Testostérone & $\begin{array}{l}\text { Epiphyse fémorale rat } \\
\text { C. Costal, rat hypox. } \\
\text { Ch. Art. lapin }\end{array}$ & $\begin{array}{l}\text { Conversion en DHT } \\
\text { Absence d'effet sur l'incorp. de } \mathrm{SO}_{4} \\
\text { Absence d'effet sur DNA, pas de stimulation } \\
\text { incorp. de } \mathrm{SO}_{4}\end{array}$ \\
\hline \multirow[t]{2}{*}{ Oestradiol } & C. Costal rat & $\begin{array}{l}\text { Absence d'effet sur incorp. de } \mathrm{SO}_{4} \text { même en } \\
\text { présence de sérum normal }\end{array}$ \\
\hline & $\begin{array}{l}\text { C. Costal porc, rat hypox. } \\
\text { C. embry. poulet } \\
\text { Ch. Art. lapin }\end{array}$ & $\begin{array}{l}\text { Absence d'effet sur l'action du sérum normal } \\
\text { Absence d'effet sur DNA }\end{array}$ \\
\hline \multirow[t]{2}{*}{ Triiodothyronine } & C. embry. poulet & $\begin{array}{l}\text { Stimule incorp. de } \mathrm{SO}_{4} \text {, effet augmenté en } \\
\text { présence de sérum et NSILA-S à des concen- } \\
\text { trations physiologiques }\end{array}$ \\
\hline & $\begin{array}{l}\text { C. épiphysaire, poulet } \\
\text { C. xyphoïde, rat }\end{array}$ & $\begin{array}{l}\text { Stimulation de l'activité phosphodiestérasique } \\
\text { Absence d'action sur l'adénylcyclase }\end{array}$ \\
\hline Dérivés de vit. $D_{3}$ & $\begin{array}{l}\text { Ch. CR. et Ch. Art. lapin et } \\
\text { Ch. CR. humain }\end{array}$ & $\begin{array}{l}\text { Stimulation de l'incorporation de leucine, } \mathrm{SO}_{4} \\
\text { par } 25(\mathrm{OH})-\mathrm{D}_{3}, 24-25(\mathrm{OH})_{2} \mathrm{D}_{3} \\
\text { Transformation de } 25(\mathrm{OH}) \mathrm{D}_{3} \text { en } 24-25(\mathrm{OH})_{2} \mathrm{D}_{3}\end{array}$ \\
\hline Cortisol & $\begin{array}{l}\text { C. Costal rat et C. embry. } \\
\text { poulet } \\
\text { C. Epiphysaire de lapin }\end{array}$ & $\begin{array}{l}\text { Inhibe l'incorp. de } \mathrm{SO}_{\dot{z}} \text { à des concentrations } \\
\text { physiologiques } \\
\text { Inhibe l'incorp. de } \mathrm{SO}_{\dot{f}} \text { ef thymidine à des } \\
\text { concentrations physiologiques }\end{array}$ \\
\hline $\begin{array}{l}\text { Parathormone et } \\
\text { Calcitonine }\end{array}$ & C. Xyphoïde, rat & Stimulation de l'activité adényl-cyclasique \\
\hline
\end{tabular}

Ch. Art. := chondrocytes articulaires.

C. embry. = cartilage embryonnaire.

Ch. CR. =- chondrocytes du cartilage de croissance.

Trois peptides isolés à partir du plasma humain se comportent comme des facteurs de croissance : NSILA-S, somatomédines $A$ et $C$ (SMA et $C$ ). Ils sont liés à un récepteur spécifique avec une haute affinité de liaison et distinct du récepteur membranaire de l'insuline. Mais ils ont aussi une certaine activité métabolique insulinique qui seraif en rapport avec une faible liaison aux récepteurs propres à l'insuline. Diverses liaisons spécifiques ont été démontrées dans le cartilage : SM A avec des membranes de chondrocytes, SM C avec des chondrocytes isolés, et des membranes de ces mêmes cellules, NSILA-S avec des chondrocytes isolés provenant de cartilage embryonnaire de poulet. Ces somatomédines et NSILA-S sont fortement liés, de manière spécifique et faiblement déplaçable par l'insuline à une protéine de transfert de PM élevé (entre 50.000 et 70.000) dont l'apparition dans le plasma serait aussi sous la dépendance de l'hormone de croissance. La preuve que ces peptides stimulent directement la croissance in vivo n'a pas encore été apportée (Chochinov et Daughaday, 1976). 
Il est possible que les autres hormones interviennent au niveau du cartilage par un effet direct propre, mais aussi en modulant la réponse du cartilage de croissance à certains de ces facteurs sériques.

Le facteur MSA (Multiplication Stimulating Activity) extrait du sérum de veau et de milieux de culture de certaines lignées de cellules hépatiques, serait très voisin des peptides précédents par son activité somatomédine et sa liaison aux récepteurs membranaires (Smith et Temin, 1974).

Les autres facteurs de croissance purifiés. Parmi les nombreux facteurs de croissance cellulaire qui ont pu être isolés, deux ont fait l'objet d'une étude sur le cartilage ; 1) le facteur de croissance de chondrocytes (CGF) (Malemud et Sokoloff, 1974) extrait de l'hypophyse humaine et contaminant des fractions TSH et $\mathrm{LH}$. Il stimule la synthèse du DNA (Corvol et al., 1972) ; 2) le facteur de croissance de fibroblastes (FGF, fibroblast growth factor) extrait du tissu cérébral ef hypophysaire de bouf, provoque aussi une forte activité mitogène sur des chondrocytes en culture (Gospodarowicz ef al., 1975).

Les stéroïdes gonadiques et surrénaliens. La testostérone est probablement métabolisée dans le cartilage en dihydrotestostérone (Jaffe et al., 1970). Aux concentrations très supraphysiologiques elle ne stimule pas la synthèse des macromolécules soufrées, ni la multiplication cellulaire (Corvol et al., 1972 ; Phillips ef al., 1975). Par contre, lorsqu'elle est administrée in vivo l'activité métabolique du cartilage (incorporation de $\mathrm{SO}_{4}$ ) est augmentée (Salmon ef al., 1963). Les effets des autres androgènes, en particulier surrénaliens n'ont pas été étudiés dans ces systèmes.

L'œstradiol n'a pas d'effet sur l'incorporation du sulfate dans le cartilage costal de rat aux concentrations étudiées qui étaient élevées (17 $\beta$-œstradiol et éthinyl-œstradiol). Il faut remarquer qu'il réduit l'activité SM circulante el provoque une augmentation de la sécrétion de STH. Il n'a pas d'effet sur l'action du sérum normal in vitro dans divers types de cartilages (Phillips et al., 1975). Le progestérone ef l'œstradiol n'ont pas d'effet sur la multiplication des chondrocytes articulaires (Corvol et al., 1972).

L'hydrocortisone inhibe l'incorporation du $\mathrm{SO}_{4}$ dans le cartilage épiphysaire de lapin à des concentrations atteignant $10^{-9} \mathrm{M}$ donc voisines de la zone physiologique (Bailly du Bois et Rappaport, résultats non publiés). Des résultats similaires ont été observés sur le cartilage embryonnaire du poulet (Eisenbarth et Lebovitz, 1974 ; Tessler et Salmon, 1975).

L'absence d'effet in vivo rapportée par certains auteurs serait liée à la nalure des protocoles expérimentaux. L'effet somatomédine du plasma est aussi diminué en présence de corticoïdes (Phillips ef al., 1975). Il esł possible que l'hydrocortisone joue un rôle régulateur sur l'effet des facteurs de croissance au niveau du cartilage.

Les dérivés de la vitamine $D_{3}$, la parathormone ef la calcitonine. Après administration de soufre radioactif à des rats rachitiques, des autoradiographies de cartilage de croissance ont montré une nette diminution du marquage radioactif autour des chondrocytes de maturation. Après viłaminothérapie, le marquage augmente rapidement dans cette zone (Hjertquist, 1961 ; Mankin et Lippiello, 1969 ; Simmons et Kunin, 1971). 
Deux métabolites de la vitamine $\mathrm{D}_{3}$, le 25 hydroxycholecalciférol $\left(25-(\mathrm{OH}) \mathrm{D}_{3}\right)$ et le 24, 25 dihydroxycholecalciférol $\left(24,25-(\mathrm{OH}) \mathrm{D}_{3}\right)$ stimulent l'incorporation de ${ }^{35 \mathrm{SO}_{4}}$ dans les protéoglycannes sécrétés par les chondrocytes de cartilage de croissance en culfure (Corvol ef al., 1977). D'autre part, le 25-(OH) $\mathrm{D}_{3}$ est transformé en 24, 25 $(\mathrm{OH})_{2} D_{3}$ après incubation avec les chondrocytes en culture ainsi qu'avec des tranches de cartilage (Garabedian ef al., 1977). Il existe très peu de travaux pour les autres hormones qui contrôlent si étroitement le tissu osseux. La parathormone augmente le contenu en AMP-cyclique du cartilage épiphysaire d'embryon de souris. Elle augmente ainsi que la calcitonine, l'activité adényl-cyclasique dans le cartilage hyalin de rat (Smith et al., 1976).

Les hormones thyroidiennes. La triiodothyronine $\left(\mathrm{T}_{3}\right)$ provoque une diminution de l'activité phosphodiestérasique dans le cartilage épiphysaire de poulet (Thanassi et Newcombe, 1974) mais n'aurait pas d'influence sur l'adénylcyclase (Smith et al., 1976) dans le cartilage de rat. Elle stimule directement la sulfatation des glycosaminoglycannes dans le cartilage embryonnaire de poulet. La thyroxine est dix fois moins active. Cet effet est majoré en présence de sérum humain normal, en utilisant des concentrations de $T_{3}$ dans les zones physiologiques (Audhya et Gibson 1975). La réponse du cartilage serait aussi liée à l'âge de l'animal, donc au stade de la chondrogénèse si l'on se souvient que les hormones thyroïdiennes stimulent plus la maturation cellulaire que la croissance au niveau de la métaphyse. NSILA-S et $\mathrm{T}_{3}$ ont un effet additif pour stimuler la synthèse protéique spécifique (Gibson ef al., 1976). Certains de ces résultats confirment les constatations classiques de synergie in vivo entre hormones thyroïdiennes ef somathormone.

Réunion Groupe Développement INRA/Productions animales Montpellier, 17-18 mai 1977.

Remerciements. - Ce travail a été réalisé grâce à l'aide financière du contrat no 76-70706 de la D.G.R.S.T.

\section{Références}

AUDHYA T. K., GIBSON K. D., 1975. Enhancement of somatomedin titers of normal and hypopituitary sera by addition of L-Triiodothyronine in vitro at physiological concentrations. Proc. nat. Acad. Sci., 72, 604-608.

CHOCHINOV R. H., DAUGHADAY W. H., 1976. Current concepts of somatomedin and other biologically related growth factors. Diabetes, 25, 994-1007.

CORVOL M. T., MALEMUD C. J., SOKOLOFF L., 1972. A pituitary growth promoting factor for articular chondrocytes in monolayer culture. Endocrinology, 90, 262-271.

CORVOL M. T., STANESCU V., MAROTEAUX P., 1974. Comparaison des propriétés électrophorétiques des protéoglycans sécrétés par les chondroxytes de cartilage de croissance et de cartilage articulaire de lapin en culture. C. R. Acad. Sci. Poris, Série D, 278, 2573-2575.

CORVOL M. T., DUMONTIER M. F., RAPPAPORT R., 1975. Culture of chondrocytes from the proliferative zone of epiphyseal growth plate cartilage from prepubertal rabbits. Biomedicine, 23, 101-107.

CORVOL M. T., DUMONTIER M. F., GARABEDIAN M., RAPPAPORT R., 1977. Vitamin D and cartilage : II. Biological activity of 25 hydroxycholecalciferol, and 24, 25 dihydroxycholecalciferol in cultured growth plate chondrocytes (soumis à Endocrinology). 
DAUGHADAY W. H., HALL K., RABEN M. S., SALMON W. D., VAN DEN BRANDE J. L., VAN WYK J. J., 1972. Somatomedin : a proposed designation for the « sulfation factor ». Nature, 235, 107.

EISENBARTH G. S., LEBCWITZ H. E., 1974. Isolation and characterization of a serum inhibitor of cartilage metabolism. Endocrinology, 95, 1600-1607.

GARABEDIAN M., BAILLY DU BOIS M., CORVOL, M. T., PEZANT E., BALSAN S., 1977. Vitamin D et cartilage : I. In vitro conversion by cartilage of 25 hydroxycholecalciferol into an active polar metabolite (soumis à Endocrinology).

GARLAND J. T., LOTTES M. E., KOZAK S., DAUGHADAY W. H., 1972. Stimulation of DNA synthesis in isolated chondrocytes by sulfation factor. Endocrinology, 90, 1086-1090.

GIBSON K. D., FROESCH E. R., BEN-PORATH E., SEGEN B., 1976. Vth int. Congr. Endocrinol., Hamburg, abstract $n^{0} 396$.

GOSPODAROWICZ D., RUDLAND P., LINDSTROM J., BERNIRSCHKE K., 1975. Fibroblast growth factor (FGF). Its localization, purification, mode of action, and physiological significance. Adv. Metab. Disord., 8, 302-336.

GREEN W. T. Jr., 1973. Behaviour of articular chondrocytes in cell culture. Clin. orthop., 75, 248.

GREGORY J. D., 1973. Multiple aggregations factors in cartilage profeoglycan. Biochem. J., 133, 383-386.

HARDINGHAM T. E., MUIR H., 1972. The specific interaction of hyaluronic acid with cartilage proteoglycans. Biochim. biophys. Acta, 279, 401-405.

HJERTQUIST S. O., 1961. Autoradiographic study of the epiphyseal cartilage and bone tissue in normal and rachitic rats after administration of radiosulphate, Biochim. Biol. Sper., 2, 126.

JAFFE R., In WILSON J. D., GLOYNA R. E., 1970. Intranuclear metabolism of testosterone. Recent Progr. Horm. Res., 26, 330-331.

MALEMUD C. J., SOKOLOFF L., 1974 . Some biological characteristics of a pituitary growth factor (CGF) for cultured lapine articular chondrocyłes. J. Cell. Physiol. 84, 171-180.

MANKIN H. J., LIPPIELLO L. J., 1969. Nucleic acid and protein synthesis in epiphyseal plates of rachitic rats : an autoradiographic study. Bone and joint Surg., 51 A, 862-874.

PITA J. C., CUERVO L. A., MADRUGA J. E., MULLER F. J., HOWELL D. S., 1970 . Evidence for a role of proteinpolysaccharides in regulation of mineral phase separation in calcifying cartilage. J. clin. Invest., 49, 2188-2197.

PHILLIPS L. S., HERINGTON A. C., DAUGHADAY W. H., 1975. Steroid hormones effects on somatomedin. I. Somatomedin action in vitro. Endocrinology, 97, 780-786.

SALMON W. D. Jr., DAUGHADAY W. H., 1957. A hormonally controlled serum factor which stimulates sulfate incorporation by cartilage in vitro. J. Lab. clin. Med., 49, 825-836.

SALMON W. D. Jr., BOWER P. H., THOMPSON E. Y., 1963. Effect of protein anabolic steroids on sulfate incorporation by cartilage of male rats. J. Lab. clin. Med., 61, 120-128.

SIMMONS J., KUNIN A. S., 1971. RNA and mucopolysaccharide metabolism in rachitic cartilage and bone. Biochem. Biophys. Aspects, 7, 412-413.

SMITH G. L., TEMIN H. M., 1974. Purified multiplication-stimulating activity from rat liver cell conditioned medium = comparison of biological activities with calf serum, insulin and somatomedin. J. cell. Physiol., 84, 181-192.

SMITH D. M., ROTH L. M., JOHNSTON C. C. Jr., 1976. Hormonal responsiveness of adenylate cyclase activity in cartilage. Endocrinology, 98, 242-246.

THANASSI N. M., NEWCOMBE D. S., 1974. Cyclic AMP and thyroid hormone inhibition of epiphyseal cartilage cyclic $3^{\prime}, 5^{\prime}$-Nucleotide phosphodiesterase activity by L-triidothyronine. Proc. Soc. exp. Biol. Med., 147, 710-714.

TESSLER R. H., SALMON W. D., 1975. Glucocorticoid inhibition of sulfate incorporation by cartilage of normal rats. Endocrinology, 96, 898-902. 